\title{
Ant Colony Optimization Algorithm for Workforce Planning: Influence of the Evaporation Parameter
}

\author{
Stefka Fidanova \\ IICT, BAS \\ Sofia, Bulgaria \\ E-mail: stefka@parallel.bas.bg
}

\author{
Gabriel Luque \\ DLCS University of Málaga \\ 29071 Málaga, Spain \\ E-mail: gabriel@1cc.uma.es \\ Maria Ganzha \\ SRI, PAS \\ Warsaw, Poland \\ E-mail: maria.ganzha@ibspan.waw.pl
}

\author{
Olympia Roeva \\ IBPhBME, BAS \\ Sofia, Bulgaria \\ E-mail: olympia@biomed.bas.bg
}

\begin{abstract}
Optimization of the production process is important for every factory or organization. The better organization can be done by optimization of the workforce planing. The main goal is decreasing the assignment cost of the workers with the help of which, the work will be done. The problem is NP-hard, therefore it can be solved with algorithms coming from artificial intelligence. The problem is to select employers and to assign them to the jobs to be performed. The constraints of this problem are very strong and for the algorithms is difficult to find feasible solutions. We apply Ant Colony Optimization Algorithm to solve the problem. We investigate the algorithm performance according evaporation parameter. The aim is to find the best parameter setting.
\end{abstract}

Index Terms-Workforce Planning, Ant Colony Optimization, Metaheuristics, Evaporation parameter

\section{INTRODUCTION}

$\mathbf{T}$ HE WORKFORCE planning is a very important decision making problem for branches of the industry. It plays an important role in human resource management. It includes multiple level of complexity, therefore it is a hard optimization problem (NP-hard). The problem can be divided in to two parts: selection and assignment. The first part is selection of employers from the set of available workers. The second part is assignment of the selected workers to jobs, which the worker will perform. The goal is to carry out the work requirements minimizing assignment cost.

As we have noted above the problem is very hard optimization problem and is impossible to be solved with exact methods or traditional numerical methods for instances with realistic size. These types of methods can be applied only on simplified variants of the problem. A deterministic version of workforce planing problem is studied in [12], [18]. In [12] the workforce planning is reformulated as mixed integer programming. It is shown that the mixed integer program is much easier to solve the problem than the non-linear program. In [18] the model includes workers differences and the possibility of workers training and upgrading. A variant with random demands of the problem is considered in [4], [19]. Two stage program of scheduling and allocating with random demands is proposed in [4]. Other variant of the problem is to include uncertainty [13], [15], [17], [24], [25]. A lot of authors skip some of the constraints to simplify the problem. Mixed linear programming is apply in [6] and in [19] is utilized decomposition method, but for the more complex non-linear workforce planning problems, the convex methods are not applicable.

Last decade, nature-inspired metaheuristic methods receive more and more attention, because they can find close to optimal solutions even for large-scale difficult problems [2], [16], [20], [22], [23]. In the literature can be found various metaheuristic algorithms solving workforce planning problem. They include genetic algorithm [1], [14], memetic algorithm [21], scatter search [1] etc.

Ant Colony Optimization (ACO) algorithm is proved to be very effective solving various complex optimization problems [7], [11]. In our previous work [8], [9] we propose ACO algorithm for workforce planning. We have considered the variant of the workforce planning problem proposed in [1] Current paper is the continuation of [8] and further develops the ideas behind [8]. We investigate the influence of evaporation parameter on algorithm performance.The aim is to find the best parameter setting.

The rest of the paper is organized as follows. In Section 2 the mathematical description of the problem is presented. In Section 3 ACO algorithm for workforce planing problem is described. Section 4 shows computational results, comparisons and discussion. A conclusion and directions for future work are done in Section 5.

\section{Workforce Planning Problem}

In this paper we consider the workforce planning problem proposed in [1] and [10]. The set of jobs $J=\{1, \ldots, m\}$ need to be completed during a fixed period of time. The job $j$ requires $d_{j}$ hours to be finished. $I=\{1, \ldots, n\}$ is the set of workers, candidates to be assigned. Every worker must perform every of assigned to him job minimum $h_{\text {min }}$ hours can work in efficient way. The worker $i$ is available $s_{i}$ hours. 
One worker can be assigned to maximum $j_{\max }$ jobs. The set $A_{i}$ shows the jobs, that worker $i$ is qualified. Maximum $t$ workers can be assigned during the planed period, or at most $t$ workers may be selected from the set $I$ of workers. The selected workers need to be capable to complete all the jobs they are assigned. The goal is to find feasible solution, that optimizes the objective function.

The cost of assigning the worker $i$ to the job $j$ is $c_{i j}$. The mathematical model of the workforce planing problem is described as follows:

$$
\begin{gathered}
x_{i j}= \begin{cases}1 & \text { if the worker } i \text { is assigned to job } j \\
0 & \text { otherwise }\end{cases} \\
y_{i}= \begin{cases}1 & \text { if worker } i \text { is selected } \\
0 & \text { otherwise }\end{cases} \\
z_{i j}=\text { number of hours that worker } i \\
\text { is assigned to perform job } j
\end{gathered}
$$

$Q_{j}=$ set of workers qualified to perform job $j$

$$
\operatorname{Minimize} \sum_{i \in I} \sum_{j \in A_{i}} c_{i j} \cdot x_{i j}
$$

Subject to

$$
\begin{gathered}
\sum_{j \in A_{i}} z_{i j} \leq s_{i} \cdot y_{i} \quad i \in I \\
\sum_{i \in Q_{j}} z_{i j} \geq d_{j} \quad j \in J \\
\sum_{j \in A_{i}} x_{i j} \leq j_{\text {max }} \cdot y_{j} \quad i \in I \\
h_{\text {min }} \cdot x_{i j} \leq z_{i j} \leq s_{i} \cdot x_{i j} \quad i \in I, j \in A_{i} \\
\sum_{i \in I} y_{i} \leq t \\
x_{i j} \in\{0,1\} \quad i \in I, j \in A_{i} \\
y_{i} \in\{0,1\} \quad i \in I \\
z_{i j} \geq 0 \quad i \in I, j \in A_{i}
\end{gathered}
$$

The objective function is the minimization of the total assignment cost. The number of hours for each selected worker is limited (inequality 2). The work must be done in full (inequality 3 ). The number of the jobs, that every worker can perform is limited (inequality 4). There is minimal number of hours that every job must be performed by every assigned worker can work efficiently (inequality 5). The number of assigned workers is limited (inequality 6).

This mathematical model can be used with other objectives too. If $\tilde{c}_{i j}$ is the cost the worker $i$ to performs the job $j$ for one hour, than the objective function can minimize the cost of the hall jobs to be finished.

$$
f(x)=\operatorname{Min} \sum_{i \in I} \sum_{j \in A_{i}} \tilde{c}_{i j} \cdot x_{i j}
$$

The preferences of the workers to the jobs can be included. In this case one of the variants of the objective function will be to maximize the satisfaction of the workers preferences.

\section{ANT COLONY OptimizATION ALGORITHM}

The ACO is a nature inspired methodology. It is a metaheuristics, following the real ants behavior when looking for a food. Real ants use chemical substance, called pheromone, to mark their path ant can return back. An ant moves in random way and when it detects a previously laid pheromone it decides whether to follow it and reinforce it with a new added pheromone. Thus the more ants follow a given trail, the more attractive that trail becomes. There is evaporation in a nature and the pheromone evaporates during the time. Thus the pheromone level of not used and less used paths decreases and they become less desirable. In this way the nature prevents the ants to follow some wrong and useless path. The ants can find a shorter path between the source of the food and the nest by their collective intelligence.

\section{A. Main ACO Algorithm}

It is not practical to solve HP-hard problems with exact methods or traditional numerical methods when the problem is large. An option is to be applied some metaheuristics. The goal is to find a good solution for a reasonable computational resources like time and memory [5].

For a first time, ant behavior is used for solving optimization problems by Marco Dorigo [3]. Later some modifications are proposed, mainly in pheromone updating rules [5]. The basic in ACO methodology is the simulation of ants behavior. The problem is represented by graph. The solutions are represented by paths in a graph and the aim is to find shorter path corresponding to given constraints. The requirements of ACO algorithm are as follows:

- Appropriate representation of the problem by a graph;

- Appropriate pheromone placement on the nodes or on the arcs of the graph;

- Suitable problem-dependent heuristic function, which manage the ants to improve solutions;

- Pheromone updating rules;

- Transition probability rule, which specifies how to include new nodes in the partial solution;

- Appropriate algorithm parameters.

The transition probability $P_{i, j}$, is a product of the heuristic information $\eta_{i, j}$ and the pheromone trail level $\tau_{i, j}$ related to the move from node $i$ to the node $j$, where $i, j=1, \ldots, n$.

$$
P_{i, j}=\frac{\tau_{i, j}^{a} \cdot \eta_{i, j}^{b}}{\sum_{k \in \text { Unused }} \tau_{i, k}^{a} \cdot \eta_{i, k}^{b}},
$$

where Unused is the set of unused nodes of the graph.

The initial pheromone level is the same for all elements of the graph and is set to a positive constant value $\tau_{0}, 0<\tau_{0}<1$. 
After that at the end of the current iteration the ants update the pheromone level [5]. A node become more desirable if it accumulates more pheromone.

The main update rule for the pheromone is:

$$
\tau_{i, j} \leftarrow \rho \cdot \tau_{i, j}+\Delta \tau_{i, j},
$$

where $\rho$ decreases the value of the pheromone, which mimics evaporation in a nature. $\Delta \tau_{i, j}$ is a new added pheromone, which is proportional to the quality of the solution. For measurement of the quality of the solution is used the value of the objective function of the ants solution.

The first node of the solution is randomly chosen. With the random start the search process is diversifying and the number of ants may be small according the number of the nodes of the graph and according other population based metaheuristic methods. The heuristic information represents the prior knowledge of the problem, which is used to better manage the algorithm performance. The pheromone is a global history of the ants to find optimal solution. It is a tool for concentration of the search around best so far solutions.

\section{B. Workforce Planing ACO}

An important role for the successes of the ACO algorithm is the representation of the problem by graph. The graph we propose is 3 dimensional and the node $(i, j, z)$ corresponds to worker $i$ to be assigned to the job $j$ for time $z$. When an ant begins their tour we generate three random numbers: the first random number is from the interval $[0, \ldots, n]$ and corresponds to the worker we assign; the second random number is from the interval $[0, \ldots, m]$ and shows the job which this worker will perform. The third random number is from the interval $\left[h_{m i n}, \min \left\{d_{j}, s_{i}\right\}\right]$ and shows number of hours worker $i$ is assigned to performs job $j$. Next node is included in the solution, applying transition probability rule. We repeat this procedure till the solution is constructed.

The following heuristic information is applied:

$$
\eta_{i j l}= \begin{cases}l / c_{i j} & l=z_{i j} \\ 0 & \text { otherwise }\end{cases}
$$

By this heuristic information the most cheapest unassigned worker, is assigned as longer as possible. The node with highest probability from all possible nodes is chosen to be included in the partial solution. When there are more than one possibilities with the same probability, the next node is chosen in a random way between them.

When a new node is included we take in to account all constraints: how many workers are assigned till now; how many time slots every worker is assigned till now; how many time slots are assigned per job till now. If a move do not meets all constraints, the probability of this move is set to 0 . The solution is constructed if there are not more possibilities for including new nodes (the transition probability is 0 for all possible moves). If the constructed solution is feasible the value of the objective function is the sum of the assignment cost of the assigned workers. When the constructed solution
TABLE I: Test instances characteristics

\begin{tabular}{|l|l|}
\hline Parameters & Value \\
\hline$n$ & 20 \\
\hline$m$ & 20 \\
\hline$t$ & 10 \\
\hline$s_{i}$ & {$[50,70]$} \\
\hline$j_{\max }$ & {$[3,5]$} \\
\hline$h_{\min }$ & {$[10,15]$} \\
\hline
\end{tabular}

TABLE II: ACO parameter settings

\begin{tabular}{|l|l|}
\hline Parameters & Value \\
\hline Number of iterations & 100 \\
\hline$\rho$ & $\{0.1,0.3,0.5,0.7,0.9\}$ \\
\hline$\tau_{0}$ & 0.5 \\
\hline Number of ants & 20 \\
\hline$a$ & 1 \\
\hline$b$ & 1 \\
\hline
\end{tabular}

is not feasible, the value of the objective function is set to be equal to -1 .

New pheromone is deposited only on the elements of feasible solutions. The deposited pheromone is proportional to the reciprocal value of the objective function.

$$
\Delta \tau_{i, j}=\frac{\rho-1}{f(x)}
$$

Thus the nodes belonging to better solutions accumulate more pheromone than others and will be more attractive in the next iteration. The iteration best solution is compared with the global best solution and if on the current iteration the some of the ants achieves better solution it becomes the new global best. As end condition we use the number of iterations.

In this research we are concentrated on influence of the evaporation parameter on algorithm performance. We tested several values for this parameter and compare the number of needed iterations to find the best solution.

\section{Computational Results AND Discussion}

In this section we tested our algorithm and evaporation parameter influence on 10 structured problems. The software, which realizes the algorithm is written in $\mathrm{C}$ and is run on Pentium desktop computer at $2.8 \mathrm{GHz}$ with $4 \mathrm{~GB}$ of memory.

An artificially generated problem instances considered in [1] is used for the tests. The test instances characteristics are shown in Table I.

The parameter settings of our ACO algorithm is shown in Table II and are fixed experimentally after several runs of the algorithm.

In our previous work [8] we show that our ACO algorithm outperforms the genetic and scatter search algorithms proposed in [1]. We perform 30 independent runs with every one of the five values of the evaporation parameter, because the algorithm is stochastic and to guarantee the robustness of the 
TABLE III: Evaporation parameter ranking

\begin{tabular}{|l|c|c|c|c|c|}
\hline & $\rho=0.1$ & $\rho=0.3$ & $\rho=0.5$ & $\rho=0.7$ & $\rho=0.9$ \\
\hline first place & 3 times & 4 times & 1 times & 2 times & 0 times \\
\hline second place & 2 times & 3 times & 3 times & 2 times & 1 times \\
\hline third place & 2 times & 1 times & 3 times & 2 times & 1 times \\
\hline forth place & 2 times & 1 times & 2 times & 3 times & 2 times \\
\hline fifth plase & 1 times & 1 times & 1 times & 1 times & 6 times \\
\hline ranking & 26 & 22 & 29 & 31 & 43 \\
\hline
\end{tabular}

average results. We apply ANOVA test for statistical analysis to guarantee the significance of the difference between the average results. We compare the average number of iterations needed to find the best result for every test problem. The needed number of iterations for every test problem can be very different, because the specificity of the tests. Therefore for comparison we use ranking as more representative. The algorithm with some fixed value for evaporation is on the first place, if it achieves the best solution with less average number of iterations over 30 runs, according other values and we assign to it 1 , we assign 2 to the value on the second place, 3 to the value on the third place, 4 to the value of the forth place and 5 to the value with most number of iterations. On some cases can be assigned same numbers if the number of iterations to find the best solution is the same. We sum the ranking of the cases over all 10 test problems to find final ranking of the different values of the evaporation parameter.

Table III shows the ranking of the evaporation parameter. The less number of iterations is needed when the evaporation parameter is equal to 0.3 . In this case the algorithm is on the first place four times, on the second place is 3 times and on the third, fourth and fifth is respectively one time. The worst results are achieved when the evaporation parameter is equal to 0.9 . The results achieved when the evaporation parameter is 0.1 are a little bit worse than when the evaporation parameter is equal to 0.3 . When the value of the evaporation parameter increase the achieved results are getting worse.

\section{CONCLUSION}

In this paper we apply ACO algorithm to solve workforce planning problem. We are concentrated on the influence of the evaporation parameter on the algorithm performance, how many iterations are needed to find the best solution. We test the algorithm on 10 structured benchmark problems. The achieved results show that when the value of the evaporation parameter is small, the algorithm needs less number of iterations compared with high value of the evaporation parameter.

\section{ACKNOWLEDGMENT}

Work presented here is partially supported by the National Scientific Fund of Bulgaria under grant DFNI DN12/5 "Efficient Stochastic Methods and Algorithms for Large-Scale Problems", Grant No BG05M2OP001-1.001-0003, financed by the Science and Education for Smart Growth Operational Program and co-financed by the European Union through the
European structural and Investment funds, and by the PolishBulgarian collaborative grant "Practical aspects for sciantific computing".

\section{REFERENCES}

[1] Alba E., Luque G., Luna F., Parallel Metaheuristics for Workforce Planning, J. Mathematical Modelling and Algorithms, Vol. 6(3), Springer, 2007, 509-528.https://doi.org/10.1007/s10852-007-9058-5

[2] Albayrak G., Özdemir İ., A state of art review on metaheuristic methods in time-cost trade-off problems, International Journal of Structural and Civil Engineering Research, Vol. 6(1), 2017, 30-34. https://doi.org/10.18178/ijscer.6.1.30-34

[3] Bonabeau E., Dorigo M. and Theraulaz G., Swarm Intelligence: From Natural to Artificial Systems, New York,Oxford University Press, 1999.

[4] Campbell G., A two-stage stochastic program for scheduling and allocating cross-trained workers, J. Operational Research Society 62(6), 2011, 1038-1047. https://doi.org/10.1057/jors.2010.16

[5] Dorigo M, Stutzle T., Ant Colony Optimization, MIT Press, 2004.

[6] Easton F., Service completion estimates for cross-trained workforce schedules under uncertain attendance and demand, Production and Operational Management 23(4), 2014, 660-675. https://doi.org/10.1111/poms.12174

[7] Fidanova S., Roeva O., Paprzycki M., Gepner P., InterCriteria Analysis of ACO Start Startegies, Proceedings of the 2016 Federated Conference on Computer Science and Information Systems, 2016, 547-550. https : //doi.org/10.1007/978 - 3-319-99648-64

[8] Fidanova S., Luquq G., Roeva O., Paprzycki M., Gepner P., Ant Colony Optimization Algorithm for Workforce Planning, FedCSIS'2017, IEEE Xplorer, IEEE catalog number CFP1585N-ART, 2017, 415-419. https://doi.org/10.15439/2017F63

[9] Roeva O., Fidanova S., Luque G., Paprzycki M., Gepner P., Hybrid Ant Colony Optimization Algorithm for Workforce Planning, FedCSIS'2018, IEEE Xplorer, 2018, 233-236. https://doi.org/10.15439/2018F47

[10] Glover F., Kochenberger G., Laguna M., Wubbena, T. Selection and assignment of a skilled workforce to meet job requirements in a fixed planning period. In:MAEB'04, 2004, 636-641.

[11] Grzybowska K., Kovács, G., Sustainable Supply Chain - Supporting Tools, Proceedings of the 2014 Federated Conference on Computer Science and Information Systems, Vol. 2, 2014, 1321-1329. https://doi.org/10.15439/2014F75

[12] Hewitt M., Chacosky A., Grasman S., Thomas B., Integer programming techniques for solving non-linear workforce planning models with learning, European J of Operational Research 242(3),2015, 942-950. https://doi.org/10.1016/j.ejor.2014.10.060

[13] Hu K., Zhang X., Gen M., Jo J., A new model for single machine scheduling with uncertain processing time, J Intelligent Manufacturing, Vol 28(3), Springer, 2015, 717-725. https://doi.org/10.1007/s10845-0151033-9

[14] Li G., Jiang H., He T., A genetic algorithm-based decom position approach to solve an integrated equipment-workforceservice planning problem, Omega, Vol. 50, Elsevier, 2015, 1-17. https://doi.org/10.1016/j.omega.2014.07.003

[15] Li R., Liu G., An uncertain goal programming model for machine scheduling problem. J. Inteligent Manufacturing, Vol. 28(3), Springer, 2014, 689-694. https://doi.org/10.1007/s10845-014-0982-8

[16] Mucherino A., Fidanova S., Ganzha M., Introducing the environment in ant colony optimization, Recent Advances in Computational Optimization, Studies in Computational Intelligence, Vol. 655, 2016, 147-158. https : //doi.org/10.1007/978 - 3-319-40132-49

[17] Ning Y., Liu J., Yan L., Uncertain aggregate production planning, Soft Computing, Vol. 17(4), Springer, 2013, 617-624. https://doi.org/10.1007/s00500-012-0931-4

[18] Othman M., Bhuiyan N., Gouw G., Integrating workers' differences into workforce planning, Computers and Industrial Engineering, Vol. 63(4), 2012, 1096-1106. https://doi.org/10.1016/j.cie.2012.06.015

[19] Parisio A, Jones CN., A two-stage stochastic programming approach to employee scheduling in retail outlets with uncertain demand, Omega, Vol. 53, Elsevier, 2015, 97-103. https://doi.org/10.1016/j.omega.2015.01.003

[20] Roeva O., Atanassova V., Cuckoo search algorithm for model parameter identification, Int. J. Bioautomation, Vol. 20(4), 2016, 483-492.

[21] Soukour A., Devendeville L., Lucet C., Moukrim A., A Memetic algorithm for staff scheduling problem in airport security service, Expert Systems with Applications, Vol. 40(18), 2013, 7504-7512. https://doi.org/10.1016/j.eswa.2013.06.073 
STEFKA FIDANOVA ET AL.: ANT COLONY OPTIMIZATION ALGORITHM FOR WORKFORCE PLANNING: INFLUENCE OF THE EVAPORATION PARAMETER 181

[22] Tilahun S.L., Ngnotchouye J.M.T., Firefly algorithm for discrete optimization problems: A survey, Journal of Civil Engineering, Vol. 21(2), 2017, 535-545. https://doi.org/10.1007/s12205-017-1501-1

[23] Toimil D., Gómes A., Review of metaheuristics applied to heat exchanger network design, International Transactions in Operational Research, Vol. 24(1-2), 2017, 7-26. https://doi.org/10.1111/itor.12296

[24] Yang G., Tang W., Zhao R., An uncertain workforce planning problem with job satisfaction, Int. J. Machine Learning and Cybernetics, Springer, 2016. https://doi.org/10.1007/s13042-016-0539-6 http://rd.springer.com/article/10.1007/s13042-016-0539-6

[25] Zhou C., Tang W., Zhao R., An uncertain search model for recruitment problem with enterprise performance, J Intelligent Manufacturing, Vol. 28(3), Springer, 2014, 295-704. doi:10.1007/s10845-014-0997-1 